\title{
PERVERSE SHEAVES ON A TRIANGULATED SPACE
}

\section{A. PolishCHuK}

The goal of this note is to prove that the category of perverse sheaves constructible with respect to a triangulation is Koszul (i.e. equivalent to the category of finite-dimensional representations of a Koszul algebra). This result was conjectured by M. Vybornov in the framework of the general theory of cellular perverse sheaves due to R. MacPherson [4], [5].

\section{Extensions between simple objects}

Let $X$ be a stratified space such that any stratum $C$ is homeomorphic to an open ball and its closure $\bar{C}$ is homeomorphic to a closed ball in a compatible way. We call strata "cells". Let $p: \mathbb{Z}_{\geq 0} \rightarrow \mathbb{Z}$ be a perversity, i.e. a function such that $p(0)=0,0 \leq p(m)-p(n) \leq n-m$ for $m \leq n$. We consider the category $\mathcal{C}$ of perverse sheaves on $X$ with respect to $p$, smooth along the stratification by cells. Let $C$ be a cell in $X, i_{C}: C \rightarrow X$ be the inclusion. Let $i_{\bar{C}}: \bar{C} \rightarrow X$ be the inlcusion of the closure of $C$. The simple objects of $\mathcal{C}$ are Goresky-MacPherson extensions $P_{C} \simeq i_{C, ! *} \mathbb{Q}_{C}[-p(C)]$, where $p(C)=p(\operatorname{dim} C)$. (Note that we do not fix an object $P_{C}$ in its isomorphism class at this point, the precise choice will be made below). Let us call an integer $i>0$ of type $*$ if $p(i)=p(i-1)-1$, and of type ! otherwise (i.e. if $p(i)=p(i-1)$ ). It is convenient to assume that 0 is of both types. We say that a cell $C$ is of type $*$ (resp. of type !) if $\operatorname{dim} C$ is.

Lemma 1.1. For any cell $C$ we have $P_{C} \simeq i_{C, !} \mathbb{Q}_{C}[-p(C)]$ if $C$ is of type!, and $P_{C} \simeq i_{C, *} \mathbb{Q}_{C}[-p(C)]$ if $i$ is of type $*$. In the latter case $P_{C} \simeq i_{\bar{C}, *} \mathbb{Q}_{\bar{C}}[-p(C)]$.

Proof. This follows easily from the fact that if $j: B \rightarrow \bar{B}$ is an inclusion of the open ball into its closure, then $j_{*} \mathbb{Q}_{B} \simeq \mathbb{Q}_{\bar{B}}$.

Now we define $P_{C}:=i_{C, *} \mathbb{Q}_{C}[-p(C)]$ if $C$ is a cell of type $*, P_{C}:=$ $i_{C, !} \operatorname{or}_{C}[-p(C)]$ if $C$ is a cell of type !, where $\operatorname{or}_{C}$ is the orientation sheaf on C.

In this situation R. MacPherson defines a perverse dimension of a cell $C$ as $\delta(C)=\delta(\operatorname{dim} C)$ where $|\delta(d)|$ is the number of $i$ 's, $0<i \leq d$, which are of the same type as $d$ (in particular, $\delta(0)=0$ ), the sign of $\delta(d)$ for $d>0$ is defined by the following rule: $\delta(d)>0$ if $d$ is of type $*$, and $\delta(d)<0$ otherwise. Notice that $\delta$ maps every interval $[0, d](d \geq 0)$ to an interval of integers containing zero, and the above definition establishes a bijective correspondence between perversities and maps $\delta: \mathbb{Z}_{\geq 0} \rightarrow \mathbb{Z}$ with this property. It is easy to see from the above

Received November 27, 1996. 
definition that $\delta(d)=-p(d)$ if $d$ is of type $*$, while $\delta(d)=-d-p(d)$ if $d$ is of type !.

From now on we assume that the cell decomposition of $X$ satisfies the following condition:

- for every pair of cells $C$ and $C^{\prime}$, the intersection $\bar{C} \cap \overline{C^{\prime}}$ is either empty or homeomorphic to a closed ball.

Below we compute Ext-groups in the derived category of sheaves on $X$ between simple objects of $\mathcal{C}$.

Proposition 1.2. Let $C$ and $C^{\prime}$ be cells. Then $\operatorname{Ext}^{i}\left(P_{C}, P_{C^{\prime}}\right)=0$ unless $i=$ $\delta(C)-\delta\left(C^{\prime}\right)$. Furthermore, $\operatorname{Ext}^{*}\left(P_{C}, P_{C^{\prime}}\right)$ is non-zero precisely in one of the following cases: 1) both $C$ and $C^{\prime}$ are of *-type (resp. !-type) and $C^{\prime} \subset \bar{C}$ (resp. $\left.C \subset \overline{C^{\prime}}\right)$, 2) $C$ is of *-type, $C^{\prime}$ is of !-type and the intersection $\bar{C} \cap \overline{C^{\prime}}$ is nonempty. In these cases $\operatorname{Ext}^{*}\left(P_{C}, P_{C^{\prime}}\right)$ is one-dimensional and has a canonical generator $e_{C, C^{\prime}}$.

Proof. Let $d=\operatorname{dim} C, d^{\prime}=\operatorname{dim} C^{\prime}$. Assume first that $C$ is of type ! and $C^{\prime}$ is of type $*$. Then $P_{C}=i_{C, !}$ or $_{C}[-p(C)], P_{C^{\prime}}=i_{C^{\prime}, *} \mathbb{Q}_{C^{\prime}}\left[-p\left(C^{\prime}\right)\right]$. Hence, the Ext-groups in question vanish since $i_{C^{\prime}} i_{C^{\prime}, *}=0$ (notice that $C \neq C^{\prime}$ ).

Assume now that $C$ and $C^{\prime}$ are of type $*$. Then one computes easily that $\operatorname{Ext}^{i}\left(P_{C}, P_{C^{\prime}}\right)$ is zero unless $i=p\left(d^{\prime}\right)-p(d)$ and $C^{\prime} \subset \bar{C}$, and in the latter case it is 1-dimensional and has a canonical generator. Note that in this case $\delta(d)-\delta\left(d^{\prime}\right)=p\left(d^{\prime}\right)-p(d)$.

The case when $C$ and $C^{\prime}$ are of type! follows from the previous one by Verdier duality (note that Verdier duality acts on perverse dimension functions by $\delta \mapsto-\delta)$.

It remains to consider the case when $C$ is of type $*$, while $C^{\prime}$ is of type !. In this case

$$
\operatorname{Ext}^{i}\left(i_{\bar{C}, *} \mathbb{Q}_{\bar{C}}, i_{C^{\prime}, !} \text { or }_{C^{\prime}}\right) \simeq H^{i}\left(\bar{C}, i_{\bar{C}}^{!} i_{C^{\prime}, !} \text { or }_{C^{\prime}}\right)
$$

Let $D$ denote the Verdier duality functor. Then

$$
i_{\bar{C}}^{!} i_{C^{\prime}, !} \text { or }_{C^{\prime}} \simeq D\left(i_{\bar{C}}^{*} i_{C^{\prime}, *} \mathbb{Q}_{C^{\prime}}\left[d^{\prime}\right]\right) \simeq D\left(i_{\bar{C} \cap \overline{C^{\prime}}, *} \mathbb{Q}_{\bar{C} \cap \overline{C^{\prime}}}\left[d^{\prime}\right]\right)
$$

It follows that

$$
\operatorname{Ext}^{i}\left(i_{\bar{C}, *} \mathbb{Q}_{\bar{C}}, i_{C^{\prime}, !} \text { or }_{C^{\prime}}\right) \simeq H^{d^{\prime}-i}\left(\bar{C} \cap \overline{C^{\prime}}\right)^{*}
$$

Since $\bar{C} \cap \overline{C^{\prime}}$ is contractible or empty, we obtain that $\operatorname{Ext}^{i}\left(P_{C}, P_{C^{\prime}}\right)$ is zero unless $i=d^{\prime}+p\left(d^{\prime}\right)-p(d)$ and $\bar{C} \cap \overline{C^{\prime}} \neq \emptyset$ (and is 1-dimensional with canonical generator when non-zero). It remains to notice that in this case $\delta(d)=-p(d)$ and $\delta\left(d^{\prime}\right)=-d^{\prime}-p\left(d^{\prime}\right)$ so that $\delta(d)-\delta\left(d^{\prime}\right)=d^{\prime}+p\left(d^{\prime}\right)-p(d)$. 


\section{Composition of Ext's}

Now we want to describe explicitly the graded algebra $A=\oplus_{C, C^{\prime}} \operatorname{Ext}^{*}\left(P_{C}, P_{C^{\prime}}\right)$ (the grading on $A$ is given by the natural grading on Ext's).

Let us write $C^{\prime} \leq C$ if $\operatorname{Ext}^{*}\left(P_{C}, P_{C^{\prime}}\right) \neq 0$. Then as follows from Proposition $1.2 \leq$ is a partial order on the set of cells (perverse inclusion). Indeed, on cells of type $*$ (resp. of type !) $\leq$ coincides with the usual adjacency order (resp. inverse adjacency order). On the other hand, if $C^{\prime} \leq C$ and $C$ and $C^{\prime}$ are of different types, then $C$ is of type $*, C^{\prime}$ is of type ! and $\bar{C} \cap \overline{C^{\prime}} \neq \emptyset$. Thus, if $C_{1} \leq C_{2}$, $C_{2} \leq C_{3}$, where $C_{1}$ and $C_{2}$ are of type ! while $C_{3}$ is of type $*$, then $\overline{C_{2}} \cap \overline{C_{3}} \neq \emptyset$ and $\overline{C_{2}} \subset \overline{C_{1}}$. Hence, $\overline{C_{1}} \cap \overline{C_{3}} \neq \emptyset$ and $C_{1} \leq C_{3}$. The case when $C_{1}$ is of type !, $C_{2}$ and $C_{3}$ are of type $*$ is similar. Note that by Proposition 1.2 if $C^{\prime} \leq C$ then $\delta\left(C^{\prime}\right) \leq \delta(C)$.

Proposition 2.1. If $C^{\prime} \leq C$ and $C^{\prime \prime} \leq C^{\prime}$ then

$$
e_{C^{\prime}, C^{\prime \prime}} \circ e_{C, C^{\prime}}=e_{C, C^{\prime \prime}}
$$

where $\circ$ denotes the composition of Ext's, $e_{?, \text { ? }}$ are canonical elements defined in Proposition 1.2.

Proof. It is sufficient to consider two cases: 1) $C, C^{\prime}$ and $C^{\prime \prime}$ are of the same type, 2) $C$ is of $*$-type, $C^{\prime \prime}$ is of !-type, and $C^{\prime}=x \in X$ is a point. The former case is obvious. In the latter case we have to prove that the following composition of natural morphisms is non-zero:

$$
i_{\bar{C}, *} \mathbb{Q}_{\bar{C}} \rightarrow i_{x, *} \mathbb{Q} \rightarrow i_{C^{\prime \prime}, !} \text { or }\left[d^{\prime \prime}\right]
$$

where $d^{\prime \prime}=\operatorname{dim} C^{\prime \prime}$. By adjointness it is equivalent to non-vanishing of the composition

$$
\mathbb{Q}_{\bar{C}} \rightarrow i_{x, *} \mathbb{Q} \rightarrow i_{\bar{C}} i_{C^{\prime \prime}, !} \text { or }\left[d^{\prime \prime}\right]
$$

Let $\bar{B}:=\bar{C} \cap \overline{C^{\prime \prime}}, b=\operatorname{dim} \bar{B}$. Then as we have seen in the proof of 1.2 one has

$$
i_{\bar{C}}^{!} i_{C^{\prime \prime}, !} \text { or }\left[d^{\prime \prime}\right] \simeq i_{\bar{B}, *} D\left(\mathbb{Q}_{\bar{B}}\right) \simeq i_{\bar{B}, *} j{ }_{\text {! or }}[b]
$$

where $j: B \rightarrow \bar{B}$ is an embedding of the open ball in $\bar{B}$. Thus our statement reduces to computing the composition

$$
\mathbb{Q}_{\bar{B}} \rightarrow i_{x, *} \mathbb{Q} \stackrel{\alpha}{\rightarrow} j ! \text { or }_{B}[b] .
$$

In other words, we have to show that the morphism of cohomologies

$$
H^{0}\left(\bar{B}, i_{x, *} \mathbb{Q}\right) \rightarrow H^{0}\left(\bar{B}, j_{!} \text {or }_{B}[b]\right)
$$

induced by $\alpha$ becomes an identity when we trivialize both spaces canonically. The Verdier dual to $\alpha$ is the canonical morphism $D \alpha: j_{*} \mathbb{Q}_{B} \simeq \mathbb{Q}_{\bar{B}} \rightarrow i_{x, *} \mathbb{Q}$, hence, the above morphism of cohomologies is dual to the canonical isomorphism $H^{0}\left(\bar{B}, j_{*} \mathbb{Q}_{B}\right) \rightarrow H^{0}\left(\bar{B}, i_{x, *} \mathbb{Q}\right)$.

Corollary 2.2. The algebra $A$ is generated by the component of degree 1. 
Proof. Let first $C^{\prime} \leq C$ be of the same type, and $\delta(C)-\delta\left(C^{\prime}\right)>1$. Then there exists a number $i$ between $\operatorname{dim} C$ and $\operatorname{dim} C^{\prime}$ such that $\delta(i)=\delta(C)-1$. It is easy to see that there exists a cell $\widetilde{C}$ of dimension $i$ such that $C^{\prime} \leq \widetilde{C}$ and $\widetilde{C} \leq C$, hence, in this case we are done by induction. Now let $C$ be of $*$-type, $C^{\prime}$ be of !-type, $\overline{C^{\prime}} \cap \bar{C} \neq \emptyset$. Choose a zero-dimensional cell $C_{0} \in \overline{C^{\prime}} \cap \bar{C}$. Then $C^{\prime} \leq C_{0}$, $C_{0} \leq C$, so this case follows from the previous one and from Proposition 2.1.

For every pair of cells $C$ and $C^{\prime}$ such that $C^{\prime} \leq C$ and $\delta(C)=\delta\left(C^{\prime}\right)+1$ we have a canonical element $e_{C, C^{\prime}} \in \operatorname{Ext}^{1}\left(P_{C}, P_{C^{\prime}}\right)$. According to the above corollary these elements generate $A$. Quadratic relations between them are described as follows. Take a couple of cells $C$ and $C^{\prime}$ such that $C^{\prime} \leq C$ and $\delta(C)=\delta\left(C^{\prime}\right)+2$. Then for all cells $\widetilde{C}$ such that $C^{\prime} \leq \widetilde{C}, \widetilde{C} \leq C$, and $\delta(\widetilde{C})=\delta\left(C^{\prime}\right)+1$, the products of $e_{C, \widetilde{C}}$ and $e_{\widetilde{C}, C^{\prime}}$ coincide. These are all quadratic relations.

Proposition 2.3. Assume that the cell-decomposition of $X$ is a simplicial triangulation. Then the algebra $A$ is quadratic.

Proof. Let $A^{\prime}$ be the quadratic algebra with $A_{1}^{\prime}=A_{1}$ and $A_{2}^{\prime}=A_{2}$ (the quadratic part of $A$ ). Note that we have a decomposition $A_{i}=\oplus A_{C, C^{\prime}}$ where $C$ and $C^{\prime}$ are cells (simplices in our case), $C \leq C^{\prime}, \delta\left(C^{\prime}\right)-\delta(C)=i$, such that the only non-zero components of the multiplication $A_{i} \otimes A_{j} \rightarrow A_{i+j}$ are of the form $A_{C, C^{\prime}} \otimes A_{C^{\prime}, C^{\prime \prime}} \rightarrow A_{C, C^{\prime \prime}}$. From this we obtain the similar decomposition for $A^{\prime}$. Hence, to check that the natural morphism $A^{\prime} \rightarrow A$ is an isomorphism it suffices to check that $\operatorname{dim} A_{C, C^{\prime}}^{\prime}=1$ (since we know that $\operatorname{dim} A_{C, C^{\prime}}=1$ ) for all $C \leq C^{\prime}$. We use induction on $i=\delta\left(C^{\prime}\right)-\delta(C)$. For $i \leq 2$ there is nothing to prove so we can assume that $i>2$ and $\delta(C) \neq-1$ (otherwise, replace $\delta$ by $-\delta, \leq$ by $\geq$, and switch $C$ and $C^{\prime}$ in the argument below). By induction hypothesis, for every $\widetilde{C}$ such that $\delta(\widetilde{C})=\delta(C)+1, C \leq \widetilde{C} \leq C^{\prime}$ the space $A_{\widetilde{C}, C^{\prime}}^{\prime}$ is 1-dimensional, and we have to check that for all such $\widetilde{C}$ the products $A_{C, \widetilde{C}}^{\prime} A_{\widetilde{C}, C^{\prime}}^{\prime} \subset A_{i}^{\prime}$ are collinear. When two such cells $\widetilde{C}_{1}$ and $\widetilde{C}_{2}$ satisfy $\widetilde{C}_{i} \leq C^{\prime \prime}(i=1,2)$ for some cell $C^{\prime \prime} \leq C^{\prime}$ such that $\delta\left(C^{\prime \prime}\right)=\delta(C)+2$ then the above collinearity is immediate. Let $V$ be the set of cells $\widetilde{C}$ such that $C \leq \widetilde{C} \leq C^{\prime}, \delta(\widetilde{C})=\delta(C)+1$. Consider the graph $\Gamma$ with the set of vertices $V$ and edges between vertices corresponding to cells $\widetilde{C}_{1}$ and $\widetilde{C}_{2}$ such that $\widetilde{C}_{i} \leq C^{\prime \prime}(i=1,2)$ for some cell $C^{\prime \prime} \leq C^{\prime}$ of perverse dimension $\delta(C)+2$. It remains to prove that $\Gamma$ is connected. Since $\delta(C)$ and $\delta(C)+2$ have the same sign (we assumed that $\delta(C) \neq-1$ ) this is the question about adjacency of faces of two given dimensions in a simplex which is easy.

\section{Koszulity}

In this section we always assume that our cell decomposition is actually a simplicial triangulation. In this case we will prove the following.

Theorem 3.1. The algebra $A$ is Koszul. 
Remark. One may suppose that the algebra $A$ of a stratification is Koszul whenever the stratification is such that the intersection of any set of closed strata is contractible (or empty). We do not know how to prove this.

According to Proposition 2.1 the algebra $A$ is completely determined by the set of cells with the partial order $\leq$ defined above. Here is a precise way to formulate this.

Let $S$ be a set with a partial order $\leq$ and a weight function $w: S \rightarrow \mathbb{Z}$, such that $w(x) \leq w(y)$ for $x \leq y$. Let $k$ be a field. Let us define an associative algebra $A_{S}$ over $k$ as follows: $A_{S}=\oplus_{x \leq y} k_{x y}$ where $x, y \in S, k_{x y}=k$, the multiplication $k_{x y} \otimes k_{y^{\prime} z} \rightarrow k_{x z}$ is zero if $y \neq y^{\prime}$ and the identity if $y=y^{\prime}$. Then $A_{S}$ has a natural grading such that $\operatorname{deg} k_{x y}=w(y)-w(x)$. Now Proposition 2.1 gives a canonical isomorphism of our algebra $A$ with $A_{S}$ where $S$ is the set of cells with the partial order $\leq$ introduced above (and $k=\mathbb{Q}$ ).

Let $A$ be an associative $k$-algebra with the decomposition $A=\oplus_{x \leq y} A_{x y}$ where $x, y \in S$, such that the multiplication $A_{x y} \otimes A_{y^{\prime} z} \rightarrow A_{x z}$ is zero unless $y=y^{\prime}$ (in the terminology of [2] $A$ is an $S$-algebra). Assume also that $A_{x x}=k$. Then every left $A$-module $M$ has a decomposition $M=\oplus_{x \in S} M_{x}$ where $M_{x}=A_{x x} M$, and the only non-zero components of $A$-action on $M$ are $A_{x^{\prime} x} M_{x} \rightarrow M_{x^{\prime}}$. Simple (left) $A$-modules correspond to elements of $S$ : for every $x \in S$ we have a simple $A$-module $k_{x}$ such that $A_{x^{\prime} x^{\prime}} k_{x}=0$ unless $x^{\prime}=x, A_{x x} k_{x}$ is 1-dimensional. Let us denote by $P_{x}$ the projective cover of $k_{x}$ defined by $\left(P_{x}\right)_{y}=A_{y x}$ if $y \leq x$ and $\left(P_{x}\right)_{y}=0$ otherwise. Recall that $A$ is called Koszul if $\operatorname{Ext}_{A}^{i}\left(k_{x}, k_{y}\right)=0$ for $i \neq w(x)-w(y)$. Notice that in any case $\operatorname{Ext}^{i}\left(k_{x}, k_{y}\right)=0$ for $i>w(x)-w(y)$.

Proposition 3.2. Let $S$ be a partially ordered set with a weight function $w$ : $S \rightarrow \mathbb{Z}$ as above. Then the following conditions are equivalent:

(1) $A_{S}$ is Koszul,

(2) for every $x, y \in S$ such that $x>y$ the algebra $A_{S(x, y)}$ is Koszul where $S(x, y)=\{z \in S: x \geq z \geq y\}$.

(3) for every $x, y \in S$, such that $x>y$ consider the subset $S^{\prime}(x, y)=$ $\{z \in S: x>z>y\}$ with the induced order. Let $\left|S^{\prime}(x, y)\right|$ be its geometric relization. Then the condition is that for every $x>y$ one has $\widetilde{H}^{i}\left(\left|S^{\prime}(x, y)\right|, k\right)=0$ for $i \neq w(x)-w(y)-2$, where $\widetilde{H}$ denotes the reduced cohomology.

Proof. Consider the bar-resolution $P^{\cdot}$ of $S_{x}$ :

$$
\ldots \rightarrow \oplus_{z_{0}<z_{1}<z_{2}<x} P_{z_{0}} \rightarrow \oplus_{z_{0}<z_{1}<x} P_{z_{0}} \rightarrow \oplus_{z_{0}<x} P_{z_{0}} \rightarrow P_{x} \rightarrow 0 .
$$

Non-zero components of Hom from the $i$-th term of this complex to $S_{y}$ correspond to summands with $z_{0}=y$. Thus, $\operatorname{Hom}\left(P^{\cdot}, S_{y}\right)$ coincides with the complex computing $\widetilde{H}^{*}\left(\left|S^{\prime}(x, y)\right|, k\right)$ shifted by 2 which proves equivalence of 1$)$ and 3$)$. Equivalence of 1) and 2) follows from this. 
Example. According to Solomon-Tits theorem the geometric realization of the set of proper non-zero subspaces of $n$-dimensional vector space (ordered by inclusion) is homotopic to a bouquet of $(n-2)$-spheres. Thus, the corresponding algebra $A_{S}$ is Koszul. We do not know if the ordered set $S$ defined by a triangulation and a perversity satisfy such stronger condition (i. e. that geometric realization of any $S^{\prime}(x, y)$ is actually homotopic to a bouquet of spheres).

Below we are going to use the notion of a quadratic PBW-algebra. The reader can consult [7] for the basic facts about this notion in the case of graded algebras. The generalization of this theory to the case of $S$-algebras is straightforward (see $[6])$.

Lemma 3.3. Let $S$ be the set of subsets of $\{1,2, \ldots, n\}$ of one of the given cardinalities $0=d_{0} \leq d_{1}<d_{2}<\ldots d_{k}=n$, with the partial order given by inclusion of subsets. Then $A_{S}$ is a quadratic PBW-algebra.

Proof. Note that $A_{S}$ is quadratic as follows from the proof of Proposition 2.3 (consider this proof for simplices of the same type). For every pair $I \subset I^{\prime}$ where $|I|,\left|I^{\prime}\right| \in\left\{d_{0}, d_{1}, \ldots, d_{k}\right\}$ let $e_{I I^{\prime}}$ be the unit in $k=k_{I I^{\prime}} \subset A_{S}$. Let us consider the lexigographical order on the subsets of $\{1,2, \ldots, n\}$ of given length. Thus, if $I=\left\{i_{1} \leq i_{2} \leq \ldots \leq i_{d}\right\}, J=\left\{j_{1} \leq j_{2} \leq \ldots \leq j_{d}\right\}$ then to compare $I$ and $J$ one first compares $i_{1}$ and $j_{1}$, then $i_{2}$ and $j_{2}$, etc. Similarly one defines lexicographical order on $e_{I I^{\prime}}$ where $I$ and $I^{\prime}$ are of given cardinalities, and on the set of words $e_{I I^{\prime}} e_{I^{\prime} I^{\prime \prime}} \ldots$. Now we claim that elements $e_{I I^{\prime}}$, where $|I|=d_{i},\left|I^{\prime}\right|=d_{i+1}$ for some $i$, are PBW-generators of $A_{S}$. Indeed, normal words of length 2 in these generators have form $e_{I J} e_{J K}$ where $|I|=d_{i},|J|=d_{i+1},|K|=d_{i+2}, I \leq J \leq K$, and $J$ is the union of $K$ and $d_{i+1}-d_{i}$ first elements of $K \backslash I$. To check the PBWcondition we have to prove that there is a unique normal word $e_{I J} e_{J K} e_{K L}$ with fixed $I$ and $L$ of length 3 (where $|I|=d_{i},|J|=d_{i+1},|K|=d_{i+2},|L|=d_{i+3}$, $I \leq J \leq K \leq L)$. Being normal means that the words $e_{I J} e_{J K}$ and $e_{J K} e_{K L}$ are normal words of length 2 . Thus, we have to prove that there is a unique sequence $I \leq J \leq K \leq L$ with fixed ends, such that $J$ is the union of $I$ and $a=d_{i+1}-d_{i}$ first elements of $K \backslash I, K$ is the union of $J$ and $b=d_{i+2}-d_{i+1}$ first elements of $L \backslash J$. It is sufficient to check that in this situation $J$ is the union of $I$ and $a$ minimal elements of $L \backslash I$. Let us prove by induction in $j=0,1, \ldots, a$ that $j$ first elements of $L \backslash I$ are contained in $J$. The base of induction $j=0$ is obvious. Assume that this is true for some $j<a$, and let $x$ be the $(j+1)$-th element of $L \backslash I$. Assume that $x \notin J$, then $x$ is the minimal element of $L \backslash J$ (since all the previous elements of $L \backslash I$ belong to $J$ by induction assumption), therefore, $x$ belongs to $K$ (since $b>0$ ). Hence, $x$ is one of the first $a$ elements of $K \backslash I$ (since $j+1 \leq a$ ) and $x$ should belong to $J$ which is a contradiction.

Lemma 3.4. Let $C$ and $C^{\prime}$ be simplices of type $*$ and !. Let $S=S_{C, C^{\prime}}$ be the partially ordered set of simplices $\widetilde{C}$ (of both types) such that $C>\widetilde{C}>C^{\prime}$. Then 
its geometric realization $|S|$ is contractible.

Proof. In this proof we consider only closed simplices. For every vertex $x \in$ $C \cap C^{\prime}$ consider the subset $S_{x} \subset S$ consisting of simplices $\widetilde{C}$ containing $x$. Then $|S|=\cup_{x}\left|S_{x}\right|$ and it is sufficient to prove that for every subset $I$ of vertices of $C \cap C^{\prime}$ the intersection $\cap_{x \in I}\left|S_{x}\right|=\left|\cap_{x \in I} S_{x}\right|$ is contractible and non-empty. But $S_{I}=\cap_{x \in I} S_{x}$ consists of all simplices $\widetilde{C} \in S$ containing the simplex $\langle I\rangle$ generated by $I$ in $C \cap C^{\prime}$. Therefore, $S_{I}$ contains an element $u$ (the simplex $\langle I\rangle$ ) such that for every other element $x \in S_{I}$ one has either $x<u$ or $x>u$. It is easy to see that this implies contractibility of $\left|S_{I}\right|$.

Proof of Theorem 3.1. According to Proposition 3.2 we have to check Koszulity of $A_{S\left(C, C^{\prime}\right)}$ for every pair of simplices $C>C^{\prime}$. When $C$ and $C^{\prime}$ are of the same type this follows from Lemma 3.3 since any PBW-algebra is Koszul. When $C$ and $C^{\prime}$ are of different types this follows from Proposition 3.2 and Lemma 3.4.

\section{Category of perverse sheaves}

In this section we derive formal consequences of the above computations.

Theorem 4.1. The category $\mathcal{C}$ is equivalent to the category of finite-dimensional representations of a quadratic algebra $B$.

Proof. The fact that $\operatorname{Ext}_{\mathcal{C}}^{1}\left(P_{C}, P_{C^{\prime}}\right)=0$ unless $\delta(C)=\delta\left(C^{\prime}\right)+1$, implies that every perverse sheaf $K \in \mathcal{C}$ has a canonical increasing filtration $F_{\leq t} K$ such that $F_{\leq t} K$ is obtained by successive extensions from the simple objects $P_{C}$ with $\delta(C) \leq t$ (see [1], 5.3.6). Furthermore, every morphism in $\mathcal{C}$ is stricly compatible with filtrations (in particular, the functors $K \mapsto \mathrm{Gr}_{t} K$ are exact). Thus, $K \mapsto \oplus \operatorname{Gr}_{t} K$ is a conservative exact functor from $\mathcal{C}$ to finite-dimensional vector spaces. This implies that $\mathcal{C}$ is equivalent to the category of finite-dimensinal representations of some algebra $B$. To prove that $B$ is quadratic it is sufficient to check that $\mathrm{Ext}^{2}$-groups between simple $B$-modules are generated by $\mathrm{Ext}^{1}$ 's between these modules. Let us denote $\widetilde{A}=\oplus \operatorname{Ext}_{\mathcal{C}}^{*}\left(P_{C}, P_{C^{\prime}}\right)$. Then we have a homomorphism of graded algebras $f: \widetilde{A} \rightarrow A$ which is an isomorphism on degree- 1 component and an injection on degree-2 component (this is a general fact about $t$-structures - see [3]). Since $A$ is generated by $A_{1}$ this implies that $f$ is an isomorphism on degree- 2 component, hence, $\widetilde{A}_{2}$ is generated by $\widetilde{A}_{1}$ as required.

The second assertion of the following theorem was announced by R. MacPherson $[5]$.

Theorem 4.2. Assume that the cell-stratification of $X$ is a simplicial triangulation. Then the above algebra $B$ is Koszul, and the bounded derived category $\mathcal{D}^{b}(\mathcal{C})$ is equivalent to the category $\mathcal{D}_{c}^{b}(X)$ of complexes of sheaves whose cohomologies are constructible with respect to the triangulation. 
Proof. According to [1] to prove the second assertion it is sufficient to check that Ext-groups between $K, L \in \mathcal{C}$ computed in these two categories coincide. Furthermore, it is sufficient to check this when $K$ and $L$ are simple objects. Now the algebra $A$ of Ext's in the category $\mathcal{D}_{c}^{b}(X)$ is Koszul by the result of the previous section. Since $\operatorname{Ext}^{i}$ between simple objects coincide in both categories when $i \leq 2$ it follows that $A$ is the dual quadratic algebra to the algebra $B$ introduced in Theorem 4.1. Since, $A$ is Koszul this implies that $B$ is Koszul. Therefore, the algebra $\widetilde{A}$ of Ioneda Ext's between simple objects of $\mathcal{C}$ is isomorphic to the quadratic dual of $B$ which is $A$.

The functor from $\mathcal{C}$ to the category of vector spaces corresponding to the equivalence of Theorem 4.1 has a very nice geometric interpretation. Let us consider the barycentric subdivision of our cell decomposition (one only needs that the closure of each cell is homeomorphic to the closed ball to define it). Now following R. MacPherson for a given cell $C$ we denote by ${ }^{\delta} C$ the union of all simplices of the barycentric subdivision with vertices $C_{1} \subset C_{2} \subset \ldots C_{k}$ such that $\delta\left(C_{i}\right) \leq \delta(C)$ for all $i$, and there exists an $i$ such that $C_{i}=C$. The subsets ${ }^{\delta} C$ are called perverse cells. Now the following theorem implies that the equivalence of Theorem 4.1 is given by the functor which sends a perverse sheaf $A$ to $\oplus_{C} H_{\delta}^{*}(X, A)$ (cohomologies with support in perverse cells).

Theorem 4.3. Let $C$ and $C^{\prime}$ be cells. Then $H_{\delta}^{*}\left(X, P_{C^{\prime}}\right)=0$ unless $C=C^{\prime}$, and $H_{\delta}^{*}\left(X, P_{C}\right)$ is one-dimensional of degree $-\delta(C)$.

Proof. Consider at first the case when $C^{\prime}$ is of type $*$. Then it is easy to see that ${ }^{\delta} C$ doesn't intersect $C^{\prime}$ unless $C=C^{\prime}$ which implies the required vanishing. On the other hand, if $C=C^{\prime}$ is of type $*$ then $C \subset{ }^{\delta} C$, hence, $H_{\delta}^{*}\left(X, P_{C}\right)=$ $H^{*}\left(C, \mathbb{Q}_{C}[-p(C)]\right)$ is one-dimensional of degree $-\delta(C)=p(C)$.

Now assume that $C^{\prime}$ is of type !. Then by Verdier duality we have to compute $H_{c}^{*}\left({ }^{\delta} C, i_{\overline{C^{\prime}}, *} \mathbb{Q}_{\overline{C^{\prime}}}\right)$. Assume at first that $C \subset \overline{C^{\prime}}$. Let ${ }^{\delta} \bar{C}$ be the closure of ${ }^{\delta} C$, $\partial^{\delta} C={ }^{\delta} \bar{C} \backslash{ }^{\delta} C$. Then the required vanishing for $C \neq C^{\prime}$ follows from the fact that in this case the intersections ${ }^{\delta} \bar{C} \cap \overline{C^{\prime}}$ and $\partial^{\delta} C \cap \overline{C^{\prime}}$ are both contractible (and non-empty). Indeed, if we represent $\overline{C^{\prime}}$ as the geometric realization of the set of cells contained in $\overline{C^{\prime}}$ (which is precisely the barycentric subdivision of $\left.\overline{C^{\prime}}\right)$, then we get representations of these intersections as geometric realizations of some sets of cells. Now both these sets of cells have the maximal element $C^{\prime}$ which implies contractibility. Assume now that $C \not \subset \overline{C^{\prime}}$. Then it is easy to see that ${ }^{\delta} C \cap \overline{C^{\prime}}=\emptyset$, hence the required vanishing. It remains to notice that if $C=C^{\prime}$ then ${ }^{\delta} \bar{C} \cap \overline{C^{\prime}}$ is a point while $\partial^{\delta} C \cap \overline{C^{\prime}}=\emptyset$.

\section{Acknowledgements}

I am grateful to A. Beilinson and L. Positselski for useful discussions and valuable remarks. 


\section{References}

1. A. Beilinson, J. Bernstein, and P. Deligne, Faisceaux pervers, Asterisque 100 (1982), 5171.

2. A. Beilinson, V. Ginzburg, and V. Schechtman, Koszul duality, J. Geom. Phys. 5 (1988), 317-350.

3. A. Beilinson, V. Ginzburg, W. Soergel, Koszul duality patterns in representation theory, J. Amer. Math. Soc. 9 (1996), 473-527.

4. M. Goresky, R. MacPherson, M. McConnel, and M. Vybornov, Cellular perverse sheaves, (to appear).

5. R. MacPherson, Perverse sheaves, course at MIT, 1993-1994.

6. A. Polishchuk and L. Positselski, Quadratic algebras, preprint, 1996.

7. S. Priddy, Koszul resolutions, Trans. Amer. Math. Soc. 152 (1970), 39-60.

Department of Mathematics, Harvard University, Cambridge, MA 02138

E-mail address: apolish@math.harvard.edu 